\title{
Molecular characterization of virus-induced autoantibody responses
}

\author{
P Krebs*1,2, B Ludewig1,2, Metters ${ }^{1}$, Ö Türeci ${ }^{3}$ and U Sahin ${ }^{3}$
}

\author{
Address: ${ }^{1}$ Institute of Experimental Immunology, Department of Pathology, University Hospital Zürich, CH-8091 Zürich, Switzerland, ${ }^{2}$ Research \\ Department, Kantonal Hospital St. Gallen, 9007 St. Gallen, Switzerland and ${ }^{3}$ III Department of Internal Medicine, Johannes Gutenberg University \\ Mainz, D-55131 Mainz, Germany \\ Email: P Krebs* - philippe.krebs@kssg.ch \\ * Corresponding author
}

from Association for Immunotherapy of Cancer: Cancer Immunotherapy - $2^{\text {nd }}$ Annual Meeting

Mainz, Germany, 6-7 May 2004

Published: I July 2004

Received: 28 April 2004

Cancer Cell International 2004, 4(Suppl I):S59

This article is available from: http://www.cancerci.com/content/4/SI/S59

We present here a comprehensive molecular mapping of virus-induced autoimmune $B$ cell responses obtained by SEREX analysis (serological identification of recombinantly expressed antigens). Immunoscreening of cDNA expression libraries of various organs (lung, liver, and spleen) using sera from mice infected with cytopathic (vaccinia virus, VV) or non-cytopathic (lymphocytic choriomeningitis virus, LCMV) viruses revealed a broad specificity of the elicited autoantibody response. Interestingly, the majority of the identified autoantigens have been previously described as autoantigens in humans. We found that induction of virus-induced autoantibodies of the IgG class largely depends on the CD40-CD40L-mediated interaction between $\mathrm{T}$ and B cells. Furthermore, antibody titers against a number of autoantigens were comparable to the concomitantly induced antiviral antibody response. Comparison of serum reactivity against a selected panel of autoantigens after infection with $\mathrm{VV}$, LCMV or vesicular stomatitis virus showed that the different virus infections triggered distinct autoantibody responses suggesting that virus infections may leave specific "autoantibody fingerprints" in the infected host. 